\title{
An Implicit Space Mapping Technique for Component Modeling
}

\author{
Qingsha S. Cheng and John W. Bandler \\ Simulation Optimization Systems (SOS) Research Laboratory, \\ McMaster University, Hamilton, ON, Canada L8S 4K1, www.sos.mcmaster.ca \\ Tel: +19056289671 Email: bandler@mcmaster.ca
}

\begin{abstract}
We present an implicit Space Mapping (ISM) technique for modeling microwave and RF components, the first attempt to employ ISM-based surrogates specifically for modeling. The proposed technique calibrates the surrogate by mapping certain preassigned parameters. This surrogate employs linearly interpolated preassigned parameters. Using a three-section transformer, we demonstrate that our technique is simple to apply within Agilent ADS. We show that we can easily enhance an HTS filter empirical model to the accuracy of an electromagnetic model, Sonnet's em.
\end{abstract}

Index Terms-CAD, optimization, EM modeling, microstrip modeling, microwave filters, space mapping, surrogate modeling.

\section{INTRODUCTION}

Space Mapping (SM) technology [1] addresses the issue of reducing unnecessary time-consuming full-wave electromagnetic (EM) simulations of microwave structures in device modeling and design optimization.

SM assumes the existence of "fine" and "coarse" models. The "fine" model may be a CPU-intensive EM simulator that provides high accuracy. The "coarse" model is a simplified representation, typically an equivalent circuit with empirical formulas. Modeling techniques [1], space derivative mapping [2], the so-called generalized SM [3] and SM-based neuromodeling [4] exploit the computational efficiency of coarse models and the accuracy of corresponding fine models so as to realize fast, accurate (enhanced) models valid over wide parameter ranges.

The SM-based modeling [5], our previous development, was designed to satisfy the desire of engineers to set up and use SM-based models in connection with (or even within) available commercial software. A family of models was implemented entirely in the Agilent ADS [6] framework.

This work was supported in part by the Natural Sciences and Engineering Research Council of Canada under Grants OGP0007239 and STGP269889, and by Bandler Corporation.

Q.S. Cheng is with the Simulation Optimization Systems Research Laboratory, Department of Electrical and Computer Engineering, McMaster University, Hamilton, ON, Canada L8S 4K1.

J.W. Bandler is with the Simulation Optimization Systems Research Laboratory, Department of Electrical and Computer Engineering, McMaster University, Hamilton, ON, Canada L8S 4K1 and also with Bandler Corporation, Dundas, ON, Canada L9H 5E7.
The expanded SM design framework (ESMDF) [7] and implicit space mapping (ISM) [8] optimization techniques exploit preassigned parameters. In this paper, we present ISM specifically for modeling. We calibrate the mapping of the preassigned parameters simultaneously at multiple on-grid points in the design parameter space in the region of interest. We extract the mapping by matching the surrogate to the corresponding fine model data. Through the mapping, the preassigned parameters are then interpolated linearly by the design parameters. Our technique is simple to apply within Agilent ADS. We show that we can easily enhance an HTS filter empirical model to the accuracy of an EM model.

\section{IMPLICIT SPACE MAPPING MODELING}

In our approach, design parameters are linearly mapped to preassigned parameters through the mapping parameters $\boldsymbol{B}_{p}$ and $\boldsymbol{c}_{p}$. We optimize $\boldsymbol{B}_{p}$ and $\boldsymbol{c}_{p}$ (parameter extraction) to match the surrogate to given fine model data.

More generally, we consider input SM ( $\boldsymbol{B}$ and $\boldsymbol{c})$, implicit SM $\left(\boldsymbol{B}_{p}\right.$ and $\boldsymbol{c}_{p}$.) and output $\mathrm{SM}(\boldsymbol{A}$ and $\boldsymbol{d})$. The surrogate formulation is written as

$$
\boldsymbol{R}_{s}\left(\boldsymbol{x}_{f}, \boldsymbol{A}, \boldsymbol{B}, \boldsymbol{c}, \boldsymbol{B}_{p}, \boldsymbol{c}_{p}, \boldsymbol{d}\right)=\boldsymbol{A} \cdot \boldsymbol{R}_{c}\left(\boldsymbol{B} \cdot \boldsymbol{x}_{f}+\boldsymbol{c}, \boldsymbol{B}_{p} \cdot \boldsymbol{x}_{f}+\boldsymbol{c}_{p}\right)+\boldsymbol{d}
$$

with matrices $\boldsymbol{A}=\operatorname{diag}\left\{a_{1}, \ldots, a_{m}\right\}, \boldsymbol{B} \in M_{n \times n}, \boldsymbol{c} \in M_{n \times 1}, \boldsymbol{B}_{p} \in M_{n \times p}$, $\boldsymbol{c}_{p} \in M_{p \times 1}$ and $\boldsymbol{d} \in M_{m \times 1}$, where $M_{k \times l}$ denotes the set of $k \times l$ real matrices. $\boldsymbol{B}$ and $\boldsymbol{c}$ account for the input mapping, $\boldsymbol{B}_{p}$ and $\boldsymbol{c}_{p}$ account for the linear interpolation of preassigned parameters. $\boldsymbol{A}$ and $\boldsymbol{d}$ provide an output mapping [9]. $\quad \boldsymbol{R}_{c}$ represents the coarse model response vector. $\boldsymbol{x}_{f}$ denotes design parameters.

The parameter extraction procedure for $q$ training points is

$$
\begin{aligned}
& \left(\overline{\boldsymbol{A}}, \overline{\boldsymbol{B}}, \overline{\boldsymbol{c}}, \overline{\boldsymbol{B}}_{p}, \overline{\boldsymbol{c}}_{p}, \overline{\boldsymbol{d}}\right)= \\
& \arg \min _{\left(\boldsymbol{A}, \boldsymbol{B}, \boldsymbol{c}, \boldsymbol{B}_{p}, \boldsymbol{c}_{p}, \boldsymbol{d}\right)} \sum_{k=1}^{q}\left\|\boldsymbol{R}_{f}\left(\boldsymbol{x}_{f}^{(k)}\right)-\boldsymbol{R}_{s}\left(\boldsymbol{x}_{f}^{(k)}, \boldsymbol{A}, \boldsymbol{B}, \boldsymbol{c}, \boldsymbol{B}_{p}, \boldsymbol{c}_{p}, \boldsymbol{d}\right)\right\|
\end{aligned}
$$

The enhanced model (surrogate) is

$$
\boldsymbol{R}_{s}\left(\boldsymbol{x}_{f}\right)=\boldsymbol{R}_{s}\left(\boldsymbol{x}_{f}, \overline{\boldsymbol{A}}, \overline{\boldsymbol{B}}, \overline{\boldsymbol{c}}, \overline{\boldsymbol{B}}_{p}, \overline{\boldsymbol{c}}_{p}, \overline{\boldsymbol{d}}\right)
$$




\section{EXAMPLES}

\section{A. Three-Section Microstrip Transformer}

We demonstrate the ADS modeling implementation for a three-section microstrip impedance transformer [Fig. 1(a)] [10]. The fine model is a Sonnet em [11] model. The coarse model, Fig. 1(b), comprises cascaded ADS microstrip empirical models. Three MSub components (MSub1, MSub2 and Msub3) introduce the preassigned parameters $\left\{\varepsilon_{1}, h_{1}, \varepsilon_{2}\right.$, $\left.h_{2}, \varepsilon_{3}, h_{3}\right\}$, the dielectric constant and the substrate height for each microstrip line. The design parameters are $\boldsymbol{x}_{f}=\left[\begin{array}{l}W_{1} L_{1} \\ W_{2} L_{2}\end{array}\right.$ $\left.W_{2} L_{2} W_{3} L_{3}\right]^{T}$. The region of interest is $12.7 \mathrm{mil} \leq W_{1} \leq 15.5$ mil, 105 mil $\leq L_{1} \leq 129$ mil, 5 mil $\leq W_{2} \leq 6.2$ mil, 108 mil $\leq$ $L_{2} \leq 132 \mathrm{mil}, 1.5 \mathrm{mil} \leq W_{3} \leq 1.9 \mathrm{mil}$, and $109 \mathrm{mil} \leq L_{3} \leq 133$ mil. The frequency range used is $5 \mathrm{GHz}$ to $15 \mathrm{GHz}$ with a step of $1 \mathrm{GHz}$ (11 points). The reflection coefficient $S_{11}$ is used to match the responses of the ISM-based surrogate and the fine model. The number of base points in the region of interest is 13. The reference point is $\boldsymbol{x}^{0}=\left[\begin{array}{llll}14.1 & 1175.61201 .7\end{array}\right.$ $121]^{T}$. The thickness of the dielectric substrate is $0.635 \mathrm{~mm}$ (25 mil) and its relative permittivity is 9.7. The preassigned parameters are linearly associated with (mapped from) the design parameters. The approach of [5] is easily modified to implement our new mapping. To avoid tedious typing, the mapping equations can be imported from a pre-defined text file using a modified AEL program from Agilent EEsof Knowledge Center (Example ID 143047).

Table I shows a statistical analysis of the $\boldsymbol{R}_{S}$ modeling errors

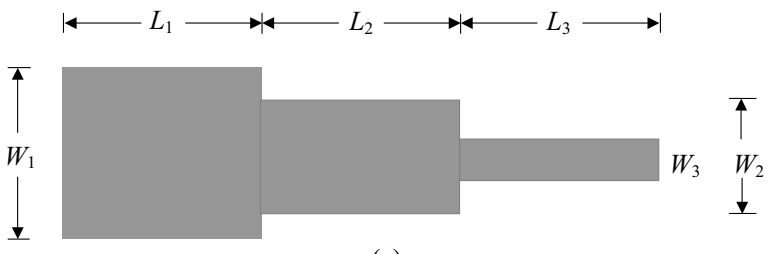

(a)

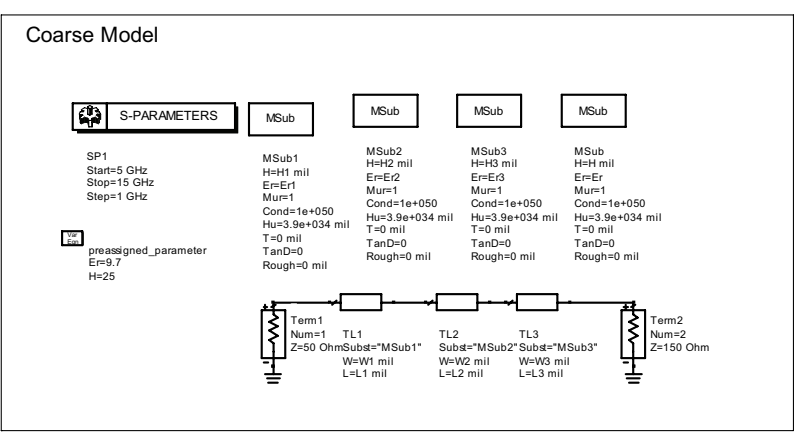

(b)

Fig. 1. The three-section 3:1 microstrip impedance transformer [10]: (a) structure and dimensions; (b) ADS coarse model.

TABLE I SM AND ISM MODELING STATISTICS FOR THREE-SECTION TRANSFORMER USING 20 RANDOM TEST POINTS

\begin{tabular}{ccc}
\hline model & mean error & maximum error \\
\hline $\boldsymbol{R}_{c}$ & 0.042 & 0.057 \\
SM-based $\boldsymbol{R}_{s}$ & 0.006 & 0.012 \\
ISM-based $\boldsymbol{R}_{s}$ & 0.004 & 0.008 \\
\hline
\end{tabular}

of 20 random test points for both SM-based and ISM-based (B $=\boldsymbol{I}$ and $\boldsymbol{c}=\mathbf{0})$ modeling. The results are both good.

\section{B. HTS Filter Example}

We consider the HTS bandpass filter of [12]. The physical structure is shown in Fig. 2(a). Design parameters are the lengths of the coupled lines and the separation between them, namely, $\boldsymbol{x}_{f}=\left[\begin{array}{llllll}S_{1} & S_{2} & S_{3} & L_{1} & L_{2} & L_{3}\end{array}\right]^{T}$. The substrate used is lanthanum aluminate with $\varepsilon_{r}=23.425, H=20 \mathrm{mil}$ and substrate dielectric loss tangent of 0.00003 . The length of the input and output lines is $L_{0}=50 \mathrm{mil}$ and the lines are of width $W=7 \mathrm{mil}$.

We use an Agilent ADS coarse model as in [8] which consists of microstrip empirical models. See Fig. 2(b). Notice the symmetry in the HTS structure, i.e., coupled lines "CLin5" are identical to "CLin1" and "CLin4" to "CLin2". We exploit different dielectric constants for each pair of coupled lines. Three ADS MSub components feed the mapped preassigned parameters (equations using the VAR component) to the coarse model. We use dielectric constants as preassigned parameters to compensate for changes in $L_{1}, L_{2}$

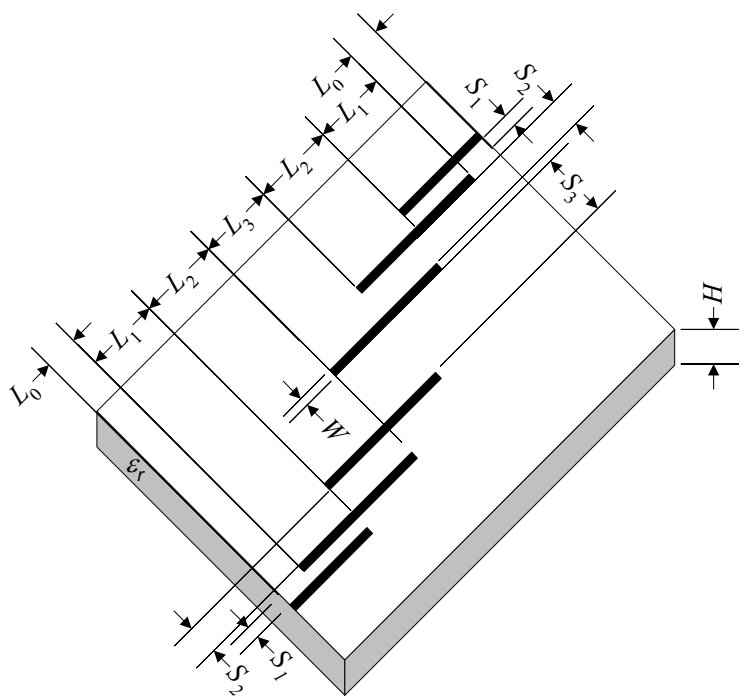

(a)

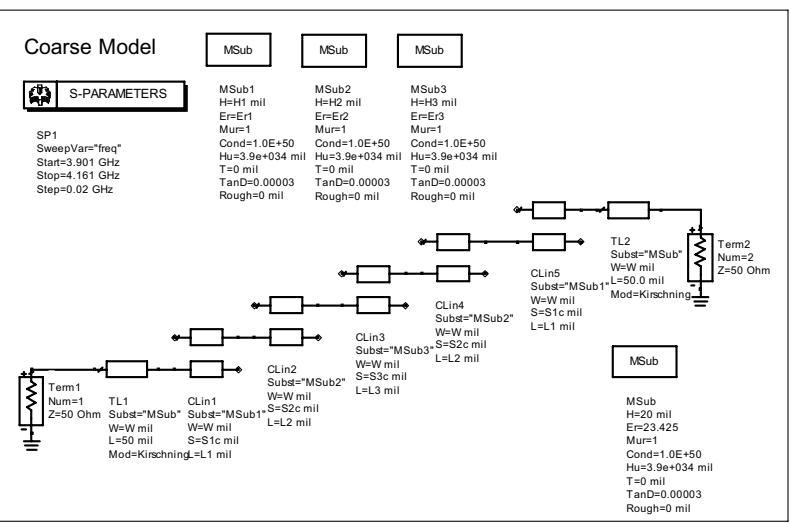

(b)

Fig. 2. The HTS filter (a) the physical structure [12], (b) the coarse model [5] as implemented in Agilent ADS. 


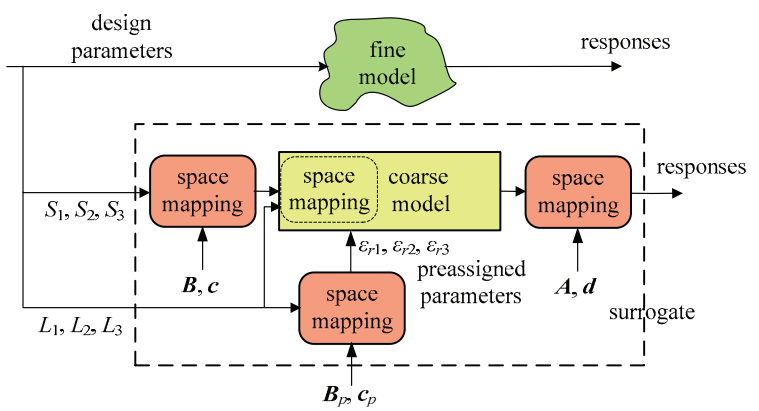

Fig. 3. ISM modeling for HTS filter configuration.

and $L_{3} . S_{1}, S_{2}$ and $S_{3}$ are still input mapped as shown in Fig. 3.

TABLE II HTS FILTER REGION OF INTEREST

\begin{tabular}{|c|c|c|c|c|c|c|}
\hline parameters & $\begin{array}{l}\text { reference } \\
\text { point }\left(x^{0}\right)\end{array}$ & $\begin{array}{c}\text { region } 1 \\
\text { size }\left( \pm \boldsymbol{\delta}_{1}\right)\end{array}$ & $\begin{array}{c}\text { region } 2 \\
\text { size }\left( \pm \boldsymbol{\delta}_{2}\right)\end{array}$ & $\begin{array}{c}\text { region } 3 \\
\text { size }\left( \pm \boldsymbol{\delta}_{3}\right)\end{array}$ & $\begin{array}{r}\text { region } 4 \\
\text { size }\left( \pm \boldsymbol{\delta}_{4}\right)\end{array}$ & $\begin{array}{c}\text { region } 5 \\
\text { size }\left( \pm \boldsymbol{\delta}_{5}\right) \\
\end{array}$ \\
\hline$L_{1}$ & 180 & 5 & 6 & 8 & 10 & 45 \\
\hline$L_{2}$ & 200 & 10 & 11 & 15 & 20 & 50 \\
\hline$L_{3}$ & 180 & 5 & 6 & 8 & 10 & 45 \\
\hline$S_{1}$ & 20 & 2 & 3 & 3 & 4 & 5 \\
\hline$S_{2}$ & 80 & 5 & 6 & 8 & 10 & 20 \\
\hline$S_{3}$ & 80 & 10 & 11 & 15 & 20 & 20 \\
\hline
\end{tabular}

The fine model is simulated by Sonnet's $\boldsymbol{e m}$ using $1 \mathrm{mil} \times$ 1 mil mesh size with 14 uniform frequency sweep points.

We use two groups of training or base points in the region of interest, the star distribution of $2 n+1$ as in [5] and the star distribution plus $2^{n}$ corner points (vertices of the region). Since we have 24 mapping parameters, the system is better determined with more base points. We test both models (with 50 points) in expanded regions. Region 1 is the original region of interest. Regions 2 through 5 are expanded as in Table II. We are interested in the $\left|S_{21}\right|$. (The wider the range the closer to zero $\left|S_{21}\right|$ becomes since the passband can shift out of the sampling band). We show selected responses comparison in Fig. 4. Error statistics w.r.t. the fine model are

\begin{tabular}{|c|c|c|c|c|c|}
\hline region & 1 & 2 & 3 & 4 & 5 \\
\hline $\boldsymbol{R}_{c}$ & 2.396 & 2.258 & 2.175 & 2.297 & 1.404 \\
\hline $\boldsymbol{R}_{s}^{\dagger}$ & 0.279 & 0.320 & 0.217 & 0.582 & 0.188 \\
\hline $\boldsymbol{R}_{s}^{\ddagger}$ & 0.220 & 0.203 & $\begin{array}{c}0.411 \\
\left(0.187^{\circ}\right)\end{array}$ & 0.275 & 0.180 \\
\hline $\boldsymbol{R}_{c}$ & 1.594 & 1.374 & 1.219 & 1.009 & 0.116 \\
\hline $\boldsymbol{R}_{s}^{\dagger}$ & 0.123 & 0.121 & 0.109 & 0.131 & 0.051 \\
\hline $\boldsymbol{R}_{s}^{\ddagger}$ & 0.114 & 0.104 & 0.095 & 0.101 & 0.028 \\
\hline
\end{tabular}

\footnotetext{
${ }^{\dagger}$ with star distribution training set

${ }^{\ddagger}$ with star distribution + corner training set

${ }^{\diamond}$ excluding one point with the largest error
}
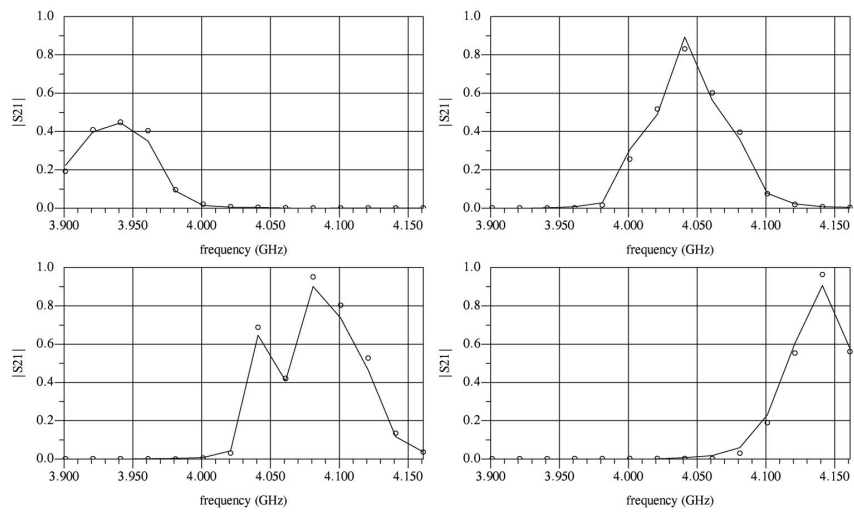

Fig. 4. HTS filter selected $\left|S_{21}\right|$ versus frequency for the fine model (o) and the $\boldsymbol{R}_{s}$ surrogate (-) in region 2.

shown in Table III for our surrogate $\boldsymbol{R}_{s}$. The model trained in the expanded base set $\left(2^{n}+2 n+1\right.$ points) outperforms the star distribution model in all 5 regions in terms of mean error and max error (except for one sample). We interpolate the new surrogate using a linear frequency interpolation technique. The surrogate is then optimized to find an optimal solution to

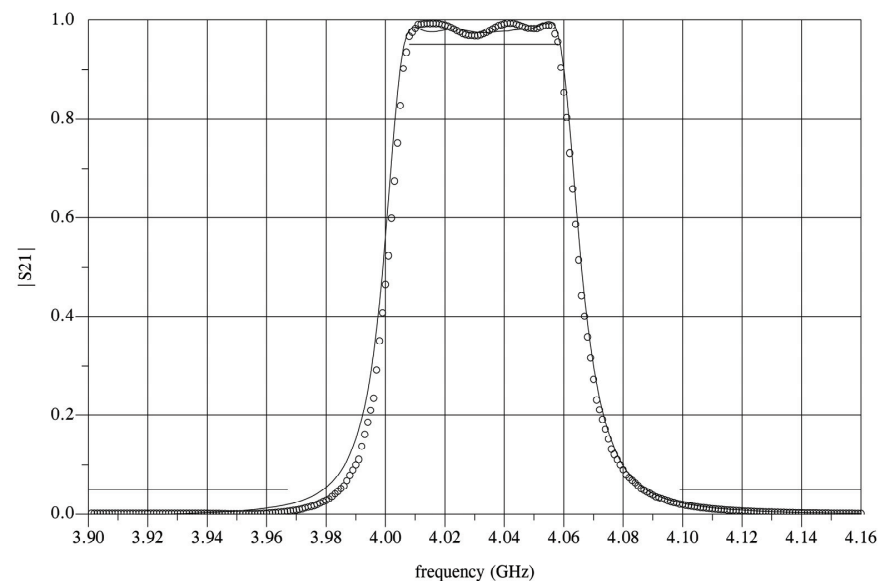

Fig. 5. HTS filter optimal $\left|S_{21}\right|$ versus frequency for the fine model (o) and the $\boldsymbol{R}_{s}$ surrogate (-) after only one surrogate model optimization.

satisfy the specification, $\left|S_{21}\right| \leq 0.05$ for $\omega \geq 4.099 \mathrm{GHz}$ and for $\omega \leq 3.967 \mathrm{GHz} ;\left|S_{21}\right| \geq 0.95$ for $4.008 \mathrm{GHz} \leq \omega \leq 4.058$ GHz.

Our design specification is satisfied in a single surrogate optimization. We verify the design using a finer frequency sweep of the fine model as shown in Fig. 5.

\section{CONCLUSIONS}

We present an effective ISM-based surrogate methodology for $\mathrm{RF}$ and microwave $\mathrm{CAD}$ modeling exploiting linearly interpolated preassigned parameters. The surrogate permits relatively small mapping matrices $\boldsymbol{B}$ or $\boldsymbol{B}_{p}$. We implement the technique in Agilent ADS in one step or one schematic. More base (training) points yield better surrogate models over extended regions. Our ISM-based surrogate provides a smooth model with high-accuracy similar to knowledge based 
neural networks [13]. It is a simple substitute for an on-grid EM simulator.

\section{ACKNOWLEDGEMENTS}

We thank Dr. S. Koziel, McMaster University, Canada, for helpful discussions. We also thank Sonnet Software, Inc., Syracuse, NY, for $\boldsymbol{e m}^{\mathrm{TM}}$ and Agilent Technologies, Santa Rosa, CA, for ADS.

\section{REFERENCES}

[1] J.W. Bandler, Q.S. Cheng, S.A. Dakroury, A.S. Mohamed, M.H. Bakr, K. Madsen and J. Søndergaard, "Space mapping: the state of the art," IEEE Trans. Microwave Theory and Tech., vol. 52, no. 1, pp. 337-361, Jan. 2004.

[2] M.H. Bakr, J.W. Bandler and N. Georgieva, "Modeling of microwave circuits exploiting space derivative mapping," IEEE MTT-S Int. Microwave Symp. Dig., Anaheim, CA, June 1999, pp. 715-718.

[3] J.W. Bandler, N. Georgieva, M.A. Ismail, J.E. Rayas-Sánchez and Q.J. Zhang, "A generalized space mapping tableau approach to device modeling," IEEE Trans. Microwave Theory Tech., vol. 49, no. 1, pp. 67-79, Jan. 2001,

[4] J.W. Bandler, M.A. Ismail, J.E. Rayas-Sánchez and Q.J. Zhang, "Neuromodeling of microwave circuits exploiting space mapping technology," IEEE Trans. Microwave Theory Tech., vol. 47, no. 12, pp. 2417-2427, Dec. 1999.
[5] Q.S. Cheng, S. Koziel and J.W. Bandler, "Simplified space-mapping approach to enhancement of microwave device models," Int. J. RF and Microwave Computer-Aided Engineering, 2006.

[6] Agilent ADS, Version 2003C, Agilent Technologies, Inc., 1400 Fountaingrove Parkway, Santa Rosa, CA 95403-1799, 2003.

[7] J.W. Bandler, M.A. Ismail and J.E. Rayas-Sánchez, "Expanded space mapping EM-based design framework exploiting preassigned parameters," IEEE Trans. Circuits and Systems-I, vol. 49, no. 12, pp. 1833-1838, Dec. 2002.

[8] J.W. Bandler, Q.S. Cheng, N.K. Nikolova and M.A. Ismail, "Implicit space mapping optimization exploiting preassigned parameters," IEEE Trans. Microwave Theory Tech., vol. 52, no. 1, pp. 378-385, Jan. 2004.

[9] S. Koziel, J.W. Bandler and K. Madsen, "Towards a rigorous formulation of the space mapping technique for engineering design," Proc. Int. Symp. Circuits Syst. ISCAS, Kobe, Japan, May 2005, pp. 5605-5608.

[10] J.W. Bandler, Q.S. Cheng, D.M. Hailu and N.K. Nikolova, "A space mapping design framework," IEEE Trans. Microwave Theory Tech., vol. 52, no. 11, pp. 2601-2610, Nov. 2004.

[11] $\boldsymbol{e m}^{\mathrm{TM}}$ Version 9.52, Sonnet Software, Inc., 100 Elwood Davis Road, North Syracuse, NY 13212, USA.

[12] J.W. Bandler, R.M. Biernacki, S.H. Chen, W.J. Getsinger, P.A. Grobelny, C. Moskowitz and S.H. Talisa, "Electromagnetic design of high-temperature superconducting microwave filters," Int. J. Microwave and Millimeter-Wave Computer-Aided Engineering, vol. 5, pp. 331-343, 1995.

[13] V.K. Devabhaktuni, B. Chattaraj, M.C.E. Yagoub and Q.J. Zhang, "Advanced microwave modeling framework exploiting automatic model generation, knowledge neural networks and space mapping," IEEE Trans. Microwave Theory Tech., vol. 51, no. 7, pp. 1822-1833, July 2003. 